\section{Methotrexate in the therapeu- tic pathway of patients with psoriasis. Analysis of clinical practice data and comparison with guidelines}

\author{
Valeria Corazza, ${ }^{1}$ Francesco Cusano, ${ }^{2}$ \\ Ornella De Pita, ${ }^{3}$ Luigi Rossi, ${ }^{4}$ \\ G. Giovanni Virno 5 \\ ${ }^{1}$ President of the Italian Psoriatic \\ Association Friends of the Corazza \\ Foundation (APIAFCO); ${ }^{2}$ President of \\ the Association of Italian Hospital \\ Dermatologists Venereologists and \\ Public Health (ADOI); ${ }^{3}$ President of the \\ International - Italian Society of Plastic- \\ Regenerative and Oncologic \\ Dermatology (ISPLAD); ${ }^{4}$ President of \\ the Confederation of Regional District \\ Associations of Tuscany Region \\ (CARD); ${ }^{5}$ Member of the Board of \\ Directors Italian Association of \\ Ambulatory Dermatologists (AIDA), \\ Italy
}

\section{Abstract}

Psoriasis is an inflammatory skin disease with a chronic-relapsing course. It is estimated that the prevalence in Italy is 3\%. An adequate model of taking care of the patient with psoriasis allows the patient to benefit from the most suitable treatment option for his health needs. In this position statement the observations, criticalities and proposals for improvement of the Pso-Path Working Group, composed by health economists, clinicians and patients, on the diagnostic-therapeutic pathway of the patient with psoriasis have been collected. In particular, the deviation of clinical practice from the current Guidelines for the management of patients with psoriasis, which recommend the use of biologic drugs in case of non-response, intolerance or contraindication to Methotrexate or Cyclosporine, was evaluated. A Working Group was convened whose participants were asked to express their thoughts on the diagnostic and therapeutic pathway of the patient with psoriasis, bringing out critical elements and proposals for improvement, based on their experiences. This position statement summarizes the experiences and consensus between clinicians and patients on actions to optimize the management of patients with psoriasis undergoing biological treatment. Compared to the epidemiological data currently available, it is believed that only a small percentage of patients with psoriasis are treated with systemic drugs. The perception of clinicians, according to their experience, confirms the data emerging from the National Report "National Observatory on the Use of Medicines" (Osmed) compiled by AIFA in 2015 , according to which more than $77 \%$ of patients with psoriasis are started to treatment with biological drugs without a previous use of Methotrexate or Cyclosporine for at least 3 months. The Pso-Path Working Group concluded that it would be desirable to incentivize, through the formalization of regional guidelines, the creation of a network system that promotes not only a greater awareness, at the territorial level, of the importance and impact of the disease and the possible paths, but also the collaboration and connection between all the actors involved in the overall care of the patient.

\section{Introduction}

Psoriasis is a chronic-recurrent inflammatory skin disease that commonly manifests itself with lesions or plaques characterized by erythema and/or desquamation. In Italy, it is estimated that about $1,800,000$ people are affected by psoriasis as the prevalence in the population is $3 \% .^{1}$ In a recent retrospective study on the administrative databases of two Italian regions, which included 8 million patients, 211,561 patients with psoriasis were identified, representing $2.6 \% .^{2}$ Moderate-severe forms affect about $1 / 4$ of patients.

The disease, because of its specifically cutaneous manifestation, which is often very visible, also has a significant impact on the quality of life of patients causing difficulties in interpersonal, social and work relationships, even reducing their selfesteem. Knees, palms of the hands, elbows, soles of the feet, scalp, genitals, trunk, are just some of the areas generally affected.

For the treatment of moderate-severe psoriasis, the use of systemic drugs is expected and, in case of ineffectiveness or intolerance, the use of biologics. However, in clinical practice the management of the pathology is rather variable.

The European guidelines "EuroGuiDerm Guideline on the systemic treatment of Psoriasis vulgaris - Part 1: treatment and monitoring recommendations - Nast et al. “, published in 2020, recommend the initiation of conventional systemic treatments as a first line of treatment, taking into account national reimbursement conditions.

In Italy, the current Guidelines of the
Correspondence: Ornella De Pità, President of the International-Italian Society of PlasticRegenerative and Oncologic Dermatology (ISPLAD), Italy.

E-mail: ornelladp@gmail.com

Key words: Psoriasis; Methotrexate; Posistion paper; Pso-Path Working Group.

Contributions: The authors contributed equally.

Conflict of interest: The authors declare no potential conflict of interest.

Funding: None.

Please cite this article as: Coraza V, Cusano $F$, De Pità $O$, et al. Methotrexate in the therapeutic pathway of patients with psoriasis. Analysis of clinical practice data and comparison with guidelines. Dermatol Rep 2022; 14:9454.

Received for publication: 28 December 2021. Accepted for publication: 28 December 2021.

This work is licensed under a Creative Commons Attribution-NonCommercial 4.0 International License (CC BY-NC 4.0).

(C) Copyright: the Author(s), 2022

Licensee PAGEPress, Italy

Dermatology Reports 2022; 14:9454

doi:10.4081/dr.2022.9454

Istituto Superiore di Sanità issued in 2013 and updated in 2016 recommend: i) the use of Methotrexate - as well as Cyclosporine and Acitretin - for the treatment of severe or plaque psoriasis; ii) the use of biologic drugs in patients with severe psoriasis who do not respond or have contraindications or are intolerant to systemic therapies.

In May 2019, in order to identify shared criteria for the appropriate use of biologic drugs, the multidisciplinary working group on Biotechnological Drugs in Dermatology of the Region of Emilia-Romagna has drawn up new Guidelines on the Treatment of Chronic Moderate-Severe Plaque Psoriasis recommending the use of bDMARDs in case of non-response, intolerance or contraindication to Methotrexate and Cyclosporine. Furthermore, it states that Methotrexate is the reference drug, among first-line drugs, for the therapy of chronic moderate-to-severe plaque psoriasis. $^{3}$

Is to be considered also the AIFA Determination No. 699 of April 15, 2019 published in G.U. No. 93 of April 19, 2019 in which it is provided that the treatment with biological drugs in NHS charge should be limited to patients with plaque psoriasis, moderate to severe grade, in case of non- 
response or intolerance (therapeutic failure) to a conventional synthetic DMARD.

Studies show that about $25-30 \%$ of patients have moderate-severe forms of psoriasis $^{1}$. It follows that in Italy about 500,000 patients are affected. A recent retrospective study on the administrative databases of two Italian regions showed that out of 211,561 patients with psoriasis, $1.1 \%$ underwent therapy with biological drugs. ${ }^{2} \mathrm{~A}$ data analysis on treatments used by patients with moderate-severe psoriasis shows that only $37,500(7 \%)$ are on systemic treatment with conventional DMARDs or biologic drugs. ${ }^{4}$ At a first reflection, it is immediately evident that, compared to the assumed epidemiology, the patients treated with systemic drugs, as required by the guidelines, are quite small.

In addition, despite the indications, the treatment path of psoriasis is not in line with the guidelines.

Data emerged in 2015 from the National Report "National Observatory on the Use of Medicines" (Osmed) prepared by AIFA. In fact, it is noted that in that year the percentage of patients with psoriasis who have started treatment with biological drugs without previous use of Methotrexate or Cyclosporine for at least 3 months was $77 \% .^{5}$

The costs analysis, subject of a recent multicentre observational study, ${ }^{6}$ also deserves some reflection. In order to verify how better prescribing of Methotrexate, as expected by the Guidelines, can have an economic impact on the National Health Service, a Budget Impact study was recently conducted, showing that increasing the use of Methotrexate by $50 \%$ (from $22.05 \%$ to $33.08 \%$ ) can reduce the overall costs of patient management. Specifically, the study showed the ability to generate national savings of 35.5 million Euros over a two-year time horizon and considering a potential population of 45,560 patients. $^{7}$

The aim of this paper is to provide insights into the psoriasis patient pathway and to collect observations, criticalities and proposals for improvement from the PsoPath Working Group, of health economists, clinicians and patients. To this end, the currently available epidemiological data will be taken into consideration, reflecting on the need for their revision and then analysing the causes of the deviation of clinical practice from the current guidelines for the management of patients with psoriasis, finally proposing initiatives for improvement to avoid inappropriate prescription.

\section{Materials and methods}

\section{The Pso-Path Table}

This document is the result of a multiprofessional discussion that has as its object the analysis of the deviation of clinical practice from the current guidelines on the care of patients with psoriasis. For this purpose, a working group, called Pso-Path, was created, composed of health economists (CliCon S.r.L.) and representatives of the Confederation of Regional District Associations (CARD), of the Association of Italian Hospital DermatologistsVenereologists and Public Health (ADOI), of the International-Italian Society of Plastic- Regenerative and Oncologic Dermatology (ISPLAD), of the Italian Association of Ambulatory Dermatologists (AIDA), of the Italian Psoriatic Association Friends of the Corazza Foundation (APIAF$\mathrm{CO})$.

The Pso-Path working group, involving scientific societies and patients on the topic of psoriasis, was motivated by the common goal of identifying areas of improvement in the current diagnostic and therapeutic setting of people with psoriasis and proposing, first of all to regional institutions, models of care and therapeutic approaches that are more effective and in line with clinical guidelines.

The Working Group discussed the results of a recent study "Analisi del percorso terapeutico nei pazienti affetti da psoriasi avviati al trattamento con farmaci biologici" carried out by CliCon, whose purpose was to analyze the patterns of conventional systemic treatments prior to biological therapy in patients with psoriasis started with biological drugs, in Italian contexts of clinical practice using real-world data. The discussion of the experts also focused on the examination of the Guidelines currently available, bringing the personal contribution derived from their experience of clinical practice.

\section{Results}

\section{Analysis of clinical practice and guidelines}

The reflections of the Pso-Path have taken into consideration first of all the epidemiological data currently available. According to these, in fact, it is estimated that in Italy there are about 1.8 million patients with psoriasis, of which about 500,000 with moderate-severe forms. We then tried to understand how these patients were treated. The data available today indicate that only 37,500 patients receive systemic treatment with conventional DMARDs or biologic drugs. Hence the need to understand what mechanisms influence the non-compliance with the Guidelines in the current diagnostic and therapeutic setting of people with psoriasis.

In addition, a temporal analysis was performed in order to measure the time interval between the use of the first-line drug and the biologic, thus investigating whether the patient's pathway to treatment with biologic is attributable to the failure of the first-line treatment or to other factors.

Recently, CliCon has carried out a retrospective observational study on the services provided and in charge of the NHS for patients diagnosed with psoriasis and started on biological therapy, between January 2013 and October 2019 (inclusion period), analysing a sample of about 3.5 million assisted (details of the methodology are reported in Section 1). The results of this analysis showed a suboptimal prescriptive appropriateness for psoriasis found in real clinical practice: in fact, of the 495 patients included with psoriasis and being treated with biological drugs, only $43.2 \%(\mathrm{~N}=214)$ had been treated with Methotrexate or Cyclosporine in the year before the start of biological therapy, and of these $52.3 \%$ $(\mathrm{N}=112)$ had received conventional treatment for a period of at least 3 months. Considering the entire population included $(\mathrm{N}=495)$, only $22.6 \%$ of patients had received Methotrexate or Cyclosporine therapy for at least 3 months in the year prior to biological treatment.

It was also found that if the whole period before the index date is considered, this percentage is reduced to 18.6 (Section 2).

Data emerged from the study were the starting point for the reflections of the working group on the actual compliance of clinical practice with the guidelines. During the discussion it emerged in fact that, according to the experience of clinicians, there is a marked variability in the management of the disease and that many patients referred directly to the treatment with biological drugs, do not follow the treatment path outlined. According to the current Guidelines of the Istituto Superiore di Sanità issued in 2013, and updated in 2016, it is recommended the use of Methotrexate - as well as Cyclosporine and Acitretin - for the treatment of severe or plaque psoriasis while the recourse to the use of biological drugs should occur for patients with severe psoriasis who do not respond or have contraindications or are intolerant to systemic therapies. According to experts' testi- 
monies, a subsequent update of the text had been hypothesized after 2016, the hypothesis, however, was not followed up due to the difficulty of bringing together all the indefectible stakeholders and for the related economic effort. It is precisely the need to identify shared criteria for the appropriate use of biologic drugs that led EmiliaRomagna Region to draw up new Guidelines on the Treatment of ModerateSevere Chronic Plaque Psoriasis. In May 2019, the multidisciplinary working group on Biotechnological Drugs in Dermatology of the Emilia- Romagna Region recommended the use of bDMARDs in case of non-response, intolerance or contraindication to Methotrexate and Cyclosporine. Moreover, it states that Methotrexate is the reference drug, among first-line drugs, for the treatment of moderate-to-severe chronic plaque psoriasis.

Moreover, the need to define a clinicaltherapeutic management process of the patient with psoriasis, which facilitates access to qualified services and appropriate care, while optimizing the use of healthcare resources, prompted Toscana Region to develop an ad hoc diagnostic-therapeutic protocol in 2014. This model of governance of the complexity of care and treatment aims to clarify the essential steps of patient management, from diagnosis to direct, continuous and planned follow-up. ${ }^{8}$

\section{Observations of the Pso-Path Table}

It is first of all perceived that the number of patients with psoriasis under treatment is considerably lower than what could be assumed considering the epidemiological data. This actually confirms the data available to date, according to which less than $10 \%$ of patients $(37,500)$ receive systemic treatment with conventional DMARDs or biological drugs. The possible reasons identified are several. First of all, in the reflections of the Pso-Path Table, the lack of a precise system for taking charge of the patient that defines the stages of the diagnostic-therapeutic pathway, identifies the points of contact and enables all the actors involved to be aligned and coordinated, has emerged with marked frequency. In the absence of such a definition, there is in fact the risk that the patient's pathway will be hindered by the absence of appropriate links between the territory and the hospital, leaving to the specialists the attempt to create them on the basis of their own knowledge rather than of a structured taking charge system.

This pathway appears to be slowed down right from the first contact with the general practitioner, in which the ability to correctly identify the symptoms and direct the patient, even in the early stages of the disease, towards the centre of reference is uncertain. All too often, in fact, the patient arrives at the specialist only at an advanced stage of the pathology, causing delays, even of years, both in the correct diagnosis and in the prescription of an appropriate treatment.

A further element brought to the attention of the members of the group concerns waiting lists, which are excessively long, pushing patients to turn to different specialists in search of adequate treatment. This continuous "search" and the relative movements can take months and, in this way, influence the timing of the treatment. This situation appears to be aggravated in the 2020s due to the recent Covid-19 pandemic, which has resulted in unprecedented difficulty in accessing treatment. In fact, missed treatment has become a real phenomenon that has focused public attention on the issue of care for non-Covid-19 patients. To take one example, according to a recent report, outpatient specialty care would have contracted from 2019 by 144.5 million fewer services, including more than 18 million visits. ${ }^{9}$

From the patients' point of view, the need for an in-depth examination of the different levels of compliance with the Guidelines at the level of individual regions emerged. The perception of the variability of treatment, which can compromise an appropriate treatment of the patient and the possibility of having access to adequate care, deserves a reflection on possible discriminatory situations that may occur due to the diversification of approaches between regions. The patient, in fact, who needs to be taken care of by a dedicated and customised treatment system based on his clinical situation, takes the risk, in the absence of a well-defined treatment pathway, of being started on treatment with a biological drug without previous use of Methotrexate, effectively skipping a therapeutic option that constitutes an additional opportunity for treatment.

The perception of clinicians, according to their experience, confirms the data emerging from the National Report "National Observatory on the Use of Medicines" (Osmed) compiled by AIFA in 2015 , according to which more than $77 \%$ of patients with psoriasis are started on treatment with biological drugs without a previous use of Methotrexate or Cyclosporine for at least 3 months. These results were also confirmed by the real- world study conducted by CliCon, which showed that about $77 \%$ of patients with psoriasis undergoing treatment with biologic drugs had not received a therapy based on Methotrexate or Cyclosporine for a duration of at least 3 months in the year preceding biological treatment (the results were detailed in Section 2). Therefore, in actual clinical practice there is a deviation from the current guidelines recommending the use of biological drugs in case of non-response, intolerance or contraindication to Methotrexate or Cyclosporine. In addition to this, there is the experience of the outpatient setting where the possibility of prescribing biological drugs depends on regional forecasts, causing a further diversification of patient management, both between regions and between local realities.

From the point of view of the economic impact on the NHS, a recent Budget Impact study showed how a $50 \%$ increase in the use of Methotrexate can reduce the overall costs of patient management, generating a saving of $€ 35.5$ million over a two-year period and considering a potential population of 45,560 patients. In these terms, the working group stressed the importance of prescribing firstline drugs identified by the Guidelines, including Methotrexate, since in the event of a patient not responding to treatment with a biologic, skipping therapy with Methotrexate corresponds to corresponds to a complete obliteration of a therapeutic opportunity with both clinical and economic consequences.

\section{Conclusions}

Currently it is estimated that in Italy there are about 1.8 million patients with psoriasis, of which about 500,000 with moderate-severe forms. Available data show that only 37,500 patients are under systemic treatment with conventional DMARDs or Biological drugs.

Based on its experience as clinicians, economists and patients, the Pso-Path Working Group first of all believes that compared to epidemiological data, the number of patients with psoriasis treated is small and further confirms that many patients diagnosed with psoriasis do not follow the treatment path outlined by the Guidelines with prior use of Methotrexate.

During the discussion, it was pointed out that there is a marked variability in the management of the disease and the need to identify shared criteria to ensure appropriate patient care in order to avoid possible discriminatory situations that may occur due to the diversification of approaches between regions. Moreover, compliance with the treatment pathway, as defined by the guidelines, is considered essential for the patient to benefit from Methotrexate as a therapeutic opportunity prior to the use of 
the biological drug. Compliance with the treatment pathway, as defined by the guidelines, also brings benefits from the point of view of the economic impact on the National Health Service. In fact, the Budget Impact study analyzed how an increase in the use of Methotrexate could reduce the overall costs of patient management, generating savings in resources that the system could use to treat new patients.

In the treatment of patients with psoriasis there are important differences between Regions and even between individual Centres. An example of this is the management of waiting lists, which, being excessively long, force the patient to turn to different specialists in search of an adequate treatment, thus delaying the timing of treatment. This situation was worsened in 2020 by the recent Covid-19 pandemic, which led to further delays and greater difficulty in accessing treatment. In fact, public attention has focused on the issue of care guaranteed to non-Covid-19 patients, and it has emerged that, compared to 2019, outpatient specialist care has suffered a reduction of 18 million visits.

In addition, there is the experience of the outpatient setting, where the possibility of prescribing biological drugs depends on regional forecasts, causing a further diversification of patient management, both between regions and between local realities.

In conclusion, in order to standardize and promote proper care of patients with psoriasis, the Pso-Path Working Group agrees on the need to encourage the creation of a network that promotes collaboration and connection among all the actors involved in the overall care of the patient. In particular, it is deemed necessary that this network system be formalized in order to define the stages of the patient's diagnostic- therapeutic pathway and facilitate communication and coordination channels between hospital and territory.

Of equal importance is the need to work on increasing awareness at the territorial level, in order to avoid situations in which the patient is referred to the specialist only at an advanced stage of the pathology, delaying, even for years, both a correct diagnosis and a prescription for appropriate treatment. This situation occurs, in fact, when symptoms are not correctly identified in the early stages of the disease and the patient's progress is slowed down from the first contact. It is therefore good to insist on the importance and impact of the pathology and the possible pathways, in order to favour the possibility of recognising the symptoms at an early stage and to start the patient on an appropriate therapeutic pathway as defined by the guidelines. It is therefore desirable to formalize regional guidelines that define the points of contact within the pathway of the patient with psoriasis that take into account local specificities, set concrete goals in terms of increasing the appropriateness of care and offer shared criteria to reduce the variability of care of patients with psoriasis.

\section{References}

1. Prignano F, Rogai V, Cavallucci E, et al. Epidemiology of Psoriasis and Psoriatic Arthritis in Italy-a Systematic Review. Curr Rheumatol Rep 2018;20:43.

2. Zagni E, Colombo D, Fiocchi M, et al. Pharmaco-utilization of biologic drugs in patients affected by psoriasis, psoriatic arthritis and ankylosing spondylitis in an Italian real-world setting. Expert Rev
Pharmacoecon Outcomes Res 2020;20: 491-7.

3. Multidisciplinary Working Group in Dermatology Emilia-Romagna Region. Systemic treatment of moderate-severe chronic plaque psoriasis with particular reference to biotechnological drugs. Therapeutic guideline no.1. Update May 2019 Assessorato Cura della persona, Salute e Welfare Regione EmiliaRomagna.

4. IQVIA Holdings Inc. IQVIA data 2nd quarter 2020. Available from: https://s24.q4cdn.com/326377938/files/ doc news/2020/07/22/IQVIA-Q22020-Earnings-Press-Release_Final.pdf

5. Agenzia Italiana del Farmaco. National Observatory for medicines use. National Report 2015. Rome: Agenzia Italiana del Farmaco, 2016.

6. Zagni E, Bianchi L, Fabbrocini G, et al. A real-world economic analysis of biologic therapies for moderate-to-severe plaque psoriasis in Italy: results of the CANOVA observational longitudinal study. BMC Health Serv Res 2021;21: 924.

7. Pompilio G, Integlia D, Aru C. Analisi di impatto sul budget SSN di una maggiore appropriatezza prescrittiva in prima linea del metotrexato nei pazienti con psoriasi di grado da moderato a severo. Available form: http://clinicoeconomics.eu/articles/flipbook/16_2 021 01-12/

8. Prignano F, Tripo L, Amato L, et al. Tuscan consensus on the diagnosis, treatment and followup of moderate-tosevere psoriasis. G Ital Dermatol Venereol 2017;152:99-108.

9. 4th Salutequità Report. Missed cures in 2020. June 2021. Available from: www.salutequita.it 